\title{
A procedure for calibrating the spinning ultrasonic wind sensors
}

\author{
M. Ghaemi-Nasab ${ }^{a, *}$, S. Franchini ${ }^{a}$, Ali R. Davari ${ }^{b}$, F. Sorribes-Palmer ${ }^{a}$ \\ a IDR/UPM, E.T.S.I. Aeronáutica y del Espacio, Universidad Politécnica de Madrid, Pza. Cardenal Cisneros, 3, E-28040 Madrid, Spain \\ ${ }^{\mathrm{b}}$ Department of Mechanical and Aerospace Engineering, Science and Research Branch, Islamic Azad University, Tehran, Iran
}

\section{A R T I C L E I N F O}

\section{Keywords:}

Spinner ultrasonic anemometer

Calibration process

Measurement uncertainties

Wind-tunnel tests

\begin{abstract}
A B S T R A C T
The accuracy of wind speed and direction measurements with spinning ultrasonic wind sensors is important than ever in today's wind industry in which, they are usually installed on the hub of the wind turbines to measure the wind speed and direction for optimized power. In this paper, extensive wind-tunnel tests have been performed to calibrate the wind speed and direction measured by a spinning single-axis ultrasonic anemometer for both static and spinning conditions. This has been carried out with static measures at various stationary angles of transducers signal path with wind direction, and with dynamic measures in which the anemometer is rotating with various rotational speeds. The velocity measured by ultrasonic anemometer in static tests is calibrated with pitottube data, and the interpolation of obtained calibration coefficients is used to correct the ultrasonic velocity measured in dynamic tests. It is observed that the calibrated ultrasonic wind speed measurements in dynamic tests are in a good agreement with the reference velocity. According to the results, the ultrasonic velocity measurements in both static and dynamic tests are affected by the transducers head distortions, and the shifting in acoustic pulse trajectory due to the rotational motion does not affect the anemometer measurements. The uncertainty of the calibration process for the spinning tests was found to be about $0.3 \%$.
\end{abstract}

\section{Introduction}

Ultrasonic anemometer, UA, is an instrument to measure the wind speed vector based on the detection of the influence of the flow field on the transmission of ultrasonic signals between a pair of facing transducers which defines its measurement path length, $L[1]$. Each transducer emits an ultrasound signal that travels towards the opposite transducer and therefore the flow velocity field has a different effect on the propagation of signals in each direction.

By measuring the differences between the forward and backward travelling times, the wind speed component along the measurement path can be determined as:

$U_{s}=\frac{L}{2}\left(\frac{1}{t_{+}}-\frac{1}{t_{-}}\right)$

This is the well-known transit-time algorithm, being used by most ultrasonic anemometers, regardless of the flow velocity field where $L$ is the length of the acoustic path, and $t_{ \pm}$is the time required by the pulse to cover the distance between transducers in sense of forward, the + subscript, and backward, the - subscript, directions [2]. This expression, however, is valid only if the velocity field is uniform.

These devices are capable of measuring multiple components of the velocity at a point. Furthermore, since there are no moving parts inside them, the ultrasonic anemometers are robust and also very suitable to use when exposed to severe weather conditions. They require very little maintenance compared to other techniques of anemometry, like hotwire, cup and propeller anemometers [3].

Altogether, the characteristics of these anemometers have made them very attractive for an extensive application range including wind energy [4], flow metering $[5,6,7,8,9]$, and urban boundary layer and atmospheric turbulent research $[10,11]$.

Recently, many investigations have raised new applications with ultrasonic anemometers installed on mobile platforms such as UAVs $[12,13]$, ships [14], atmospheric probes $[15,16]$ or wind turbines $[17,18]$. These new applications have brought the issue of studying their behaviors during motion and rotation, into forefront of the recent surveys.

In wind turbine applications, a fixed 2D sonic anemometer along with cup anemometer and wind vane, are commonly used to measure the wind speed and direction, known as nacelle anemometry. The measurements by these anemometers in nacelle anemometry are affected by the wakes and flow distortion generated by the blades and nacelle chamber [19]. A previous research by Zahle et al. [20], has shown a remarkable influence of flow distortions on the nacelle anemometers.

A recent developed technique to nacelle anemometry is spinner 


\begin{tabular}{|c|c|c|c|}
\hline \multicolumn{2}{|c|}{ Nomenclature } & $U_{s}$ & velocity measured by ultrasonic anemometer $[\mathrm{m} / \mathrm{s}]$ \\
\hline$L$ & acoustic path length between two transducers [m] & & {$[\mathrm{m} / \mathrm{s}]$} \\
\hline$D$ & diameter of ultrasonic transducers [m] & $U_{r}$ & reference velocity $[\mathrm{m} / \mathrm{s}]$ \\
\hline$t_{+}, t_{-}$ & $\begin{array}{l}\text { travelling time of the acoustic pulses in forward and } \\
\text { backward directions [sec] }\end{array}$ & $\theta$ & $\begin{array}{l}\text { azimuthal angle between transducers' signal path with } \\
\text { flow direction [rad] }\end{array}$ \\
\hline$f$ & sampling frequency $[\mathrm{Hz}]$ & $\sigma$ & standard deviation \\
\hline$\omega$ & angular velocity $[\mathrm{rad} / \mathrm{s}]$ & $C, a$ & transducer shadow effect parameters \\
\hline$U_{\infty}$ & free stream wind speed $[\mathrm{m} / \mathrm{s}]$ & $U_{n}$ & theoretical model $[\mathrm{m} / \mathrm{s}]$ \\
\hline
\end{tabular}

anemometry, which uses three single-axis ultrasonic anemometers to measure wind speed and direction over the wind turbine spinner. Therefore, the UAs in spinner anemometry are not affected by such flow distortions experienced by nacelle anemometty [21]. There are still some distortions in front of the wind turbine; of course they are less pronounced, but they still influence the measurement results and a proper calibration process to take this into account should be performed.

In a wind turbine, accurate information about the incoming wind speed and direction is important in yaw and pitch regulations for optimized wind turbine power. Furthermore, since the wind energy is directly proportional to the thitd power of the wind speed [22], an accurate wind speed measurement, as performed by a properly calibrated anemometer, would be extremely needed to achieve the maximum performance from energy saving viewpoint.

Although there are investigations in which the performance of the spinner ultrasonic anemometer is evaluated on wind turbine, $[18,23,24]$, it has not found a systematic study on the behavior of these devices in rotational conditions. According to the Standard: IEC 6140012-2 [25], the calibration process that is used for spinner anemometry consists of three major phases including the wind tunnel calibration tests for each ultrasonic sensor, the calibration of each spinner UA installations, and the calibration of each wind turbine type.

Currently the calibration process for each UA, the first phase, is a series of wind-tunnel tests in which the anemometer is calibrated for vatious stationaty inflow angles of attack such that wind direction is fixed and not changed during the test [26]. However, in the present paper, the wind speed measurements with a 1D sonic sensor, similat to ones used in spinner anemometry, are calibrated under both static and rotational conditions.

In the static tests, the anemometer's signal path was set in various stationary orientations with respect to the wind direction, and several wind speed has been examined for each test.

In the dynamic tests, the anemometer was rotating with various angular velocities, exposed to different wind speeds. Thus, a complex system was designed for supplying power and downloading the measurements from the spinning UA. The calculated correction factors from the static tests were used to correct the measurement data in the spinning cases.

Indeed, the objective of this article is to propose a calibration process for wind speed indicated by the spinning single-axis UA. The calibration process consists of two series of expetiments in an accredited wind-tunnel. Firstly, the UA was placed in various angles of attack toward the inflow direction, and its measurements were calibrated with pitot-tube. The calculated calibration coefficients were then used for correcting the wind speed measured by the same anemometer in totational motion.

\section{Experimental setup}

Experiments were petformed in the $\$ 4$ low turbulence wind tunnel of IDR institute in the Polytechnic University of Madrid, Spain. Fig. 1 shows the schematic side view of the wind tunnel which is of open circuit with a closed test section. The wind speed is controlled electronically by a vatiable frequency dive, and flow velocities can be attained up to $25 \mathrm{~m} / \mathrm{s}$ with the flow uniformity in the test section better than $0.2 \%$ and the turbulence intensity less than $0.1 \%$. This wind tunnel fulfills the requirements of the MEASNET and the ISO/IEC17025 standard which is mainly used for the calibration of anemometers.

A specially designed single-axis ultrasonic anemometer, which was a modification of the ' $\mathrm{K}$ style-probe' ultrasonic anemometer from Applied Technologies Inc., has been used for the experiments. The sonic path length ( $L$ ) of this anemometer is $0.15 \mathrm{~m}$, and it can measure wind speed in a range of $\pm 30 \mathrm{~m} / \mathrm{s}$ with a resolution of $0.01 \mathrm{~m} / \mathrm{s}$. The sonic path has been mounted on a system that allowed to totating at constant and controlled angular speed. Note that the designed system has a high complexity due to the necessity of supplying power and recover the signals measured by the UA while it is rotating.

Other than the UA itself, the main components of the installation were a DC motor and a controller for its speed, a contactless angular position sensor and a slip ring rotating connector that supplied current to the sonic path and downloaded the output signals, while the UA was rotating inside the wind tunnel test chamber. The free stream velocity was measured with a pitot tube and a pressure cap of DRUCK (model LPM 9481). Fig. 2 shows a photo of the modified UA installed in the S4 wind tunnel, and a schematic view of the installations in the test section. During the experiments, the measurements from UA and angular position sensor have been synchronized at the same time base. It is claimed that the structure is designed and constructed in a way that it has a negligible vibration during the rotational tests which cannot affect the measurements.

The static test was similar to the typical calibration procedures of an anemometer, i.e. it was subjected to the wind tunnel flow, varying the wind speed from 0 to $23 \mathrm{~m} / \mathrm{s}$, with a fixed otientation of the acoustic

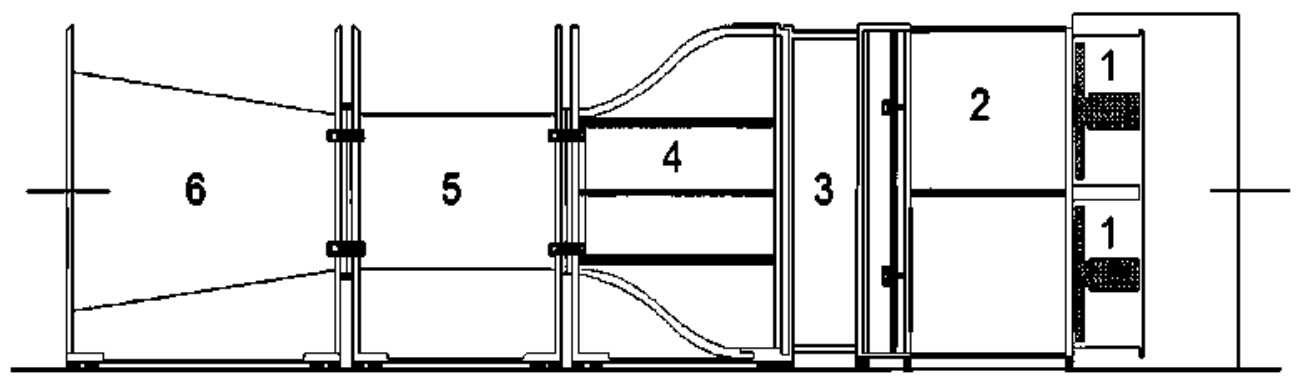

Fig. 1. Sketch of the S4 wind.tunnel: 1 = fans, 2 = plenum chamber, 3 = honeycomb and grids, $4=$ contraction, $5=$ test section, and $6=$ dif. fuser. 

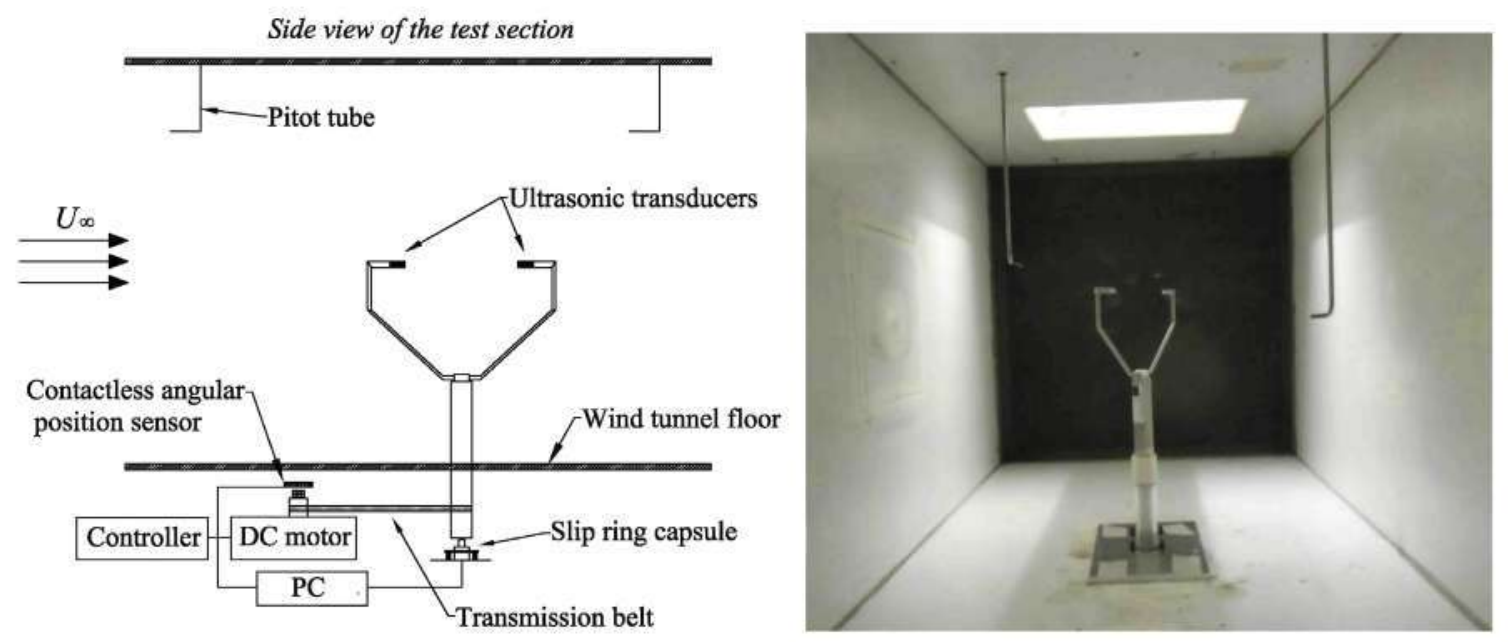

Fig. 2. Modified UA installed in the test section of the $\$ 4$ wind-tunnel.

Table 1

Tested rotational speeds of the sonic path.

\begin{tabular}{ll}
\hline Test no. & UA rotational speed, $\omega[\mathrm{rad} / \mathrm{s}]$ \\
\hline 1 & 1.11 \\
2 & 1.68 \\
3 & 3.18 \\
4 & 3.45 \\
5 & 4.64 \\
6 & 5.69 \\
7 & 6.73 \\
8 & 8.53 \\
9 & 9.71 \\
10 & 11.01 \\
11 & 13.50 \\
12 & 22.98 \\
\hline
\end{tabular}

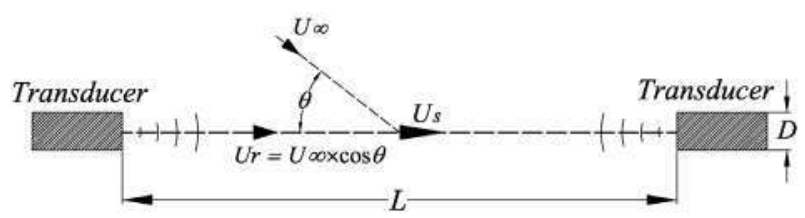

Fig. 3. Schematic configuration of the UA. $L$ is the length between transducers, $D$ is the transducers diameter, $U_{-}$is the free stream wind speed, $\theta$ is the azimuthal angle between flow direction and UA signal path, $U_{s}$ is the velocity measured by UA, and $U_{r}$ is the projection of the inflow wind component onto the UA signal path.

path, $\theta$. These tests have been repeated at 13 different values of azimuthal angle, $\theta$, between $0^{\circ}$ and $180^{\circ}$.

In the dynamic tests, a rotational speed was set with the DC motor controller, and different wind speeds were selected for the wind tunnel. At the same time, the pitot-tube readings, the instantaneous angular position and the UA output signals were recorded. This procedure has been repeated for 13 different wind speeds between $0-23 \mathrm{~m} / \mathrm{s}$ and for the 12 rotational velocities of the sonic path presented in Table 1.

The instantaneous angular positions were recorded accordingly from the angular position sensor. In both series of tests, the measures of the ultrasonic anemometer and the angular position sensor were recorded during $42 \mathrm{~s}$, at a sample rate of $50 \mathrm{~Hz}$. Also, the ambient conditions, air temperature, pressure and humidity, have been recorded during the experiments.

Fig. 3 illustrates the schematic configuration of the tested UA, indicating the transducers diameter, $D$, the acoustic path length, $L$, the free stream wind speed vector, $U_{\infty}$, the definition of the azimuthal angle between signal path with free stream wind direction, $\theta$, the velocity measured by the UA along the signal path, $U_{s}$, and the projection

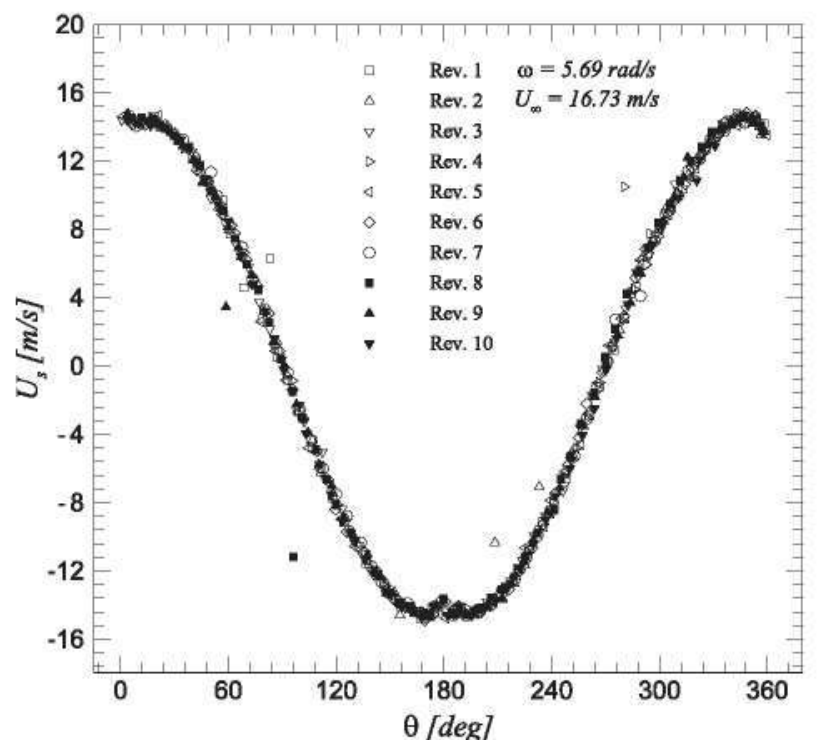

Fig. 4. UA indicated velocity at different instantaneous azimuthal angles for 10 revolutions.

Table 2

The calculated statistical parameters for the 10 revolutions.

\begin{tabular}{llc}
\hline Mean & Median & Skewness \\
\hline 0.0256 & 0.1 & -0.0105 \\
\hline
\end{tabular}

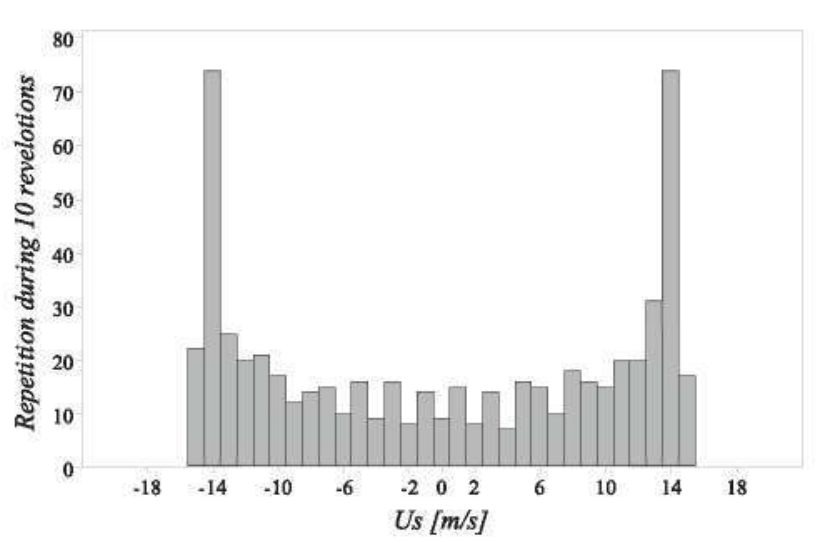

Fig. 5. Histogram of the UA velocity measurements during 10 revolutions. 


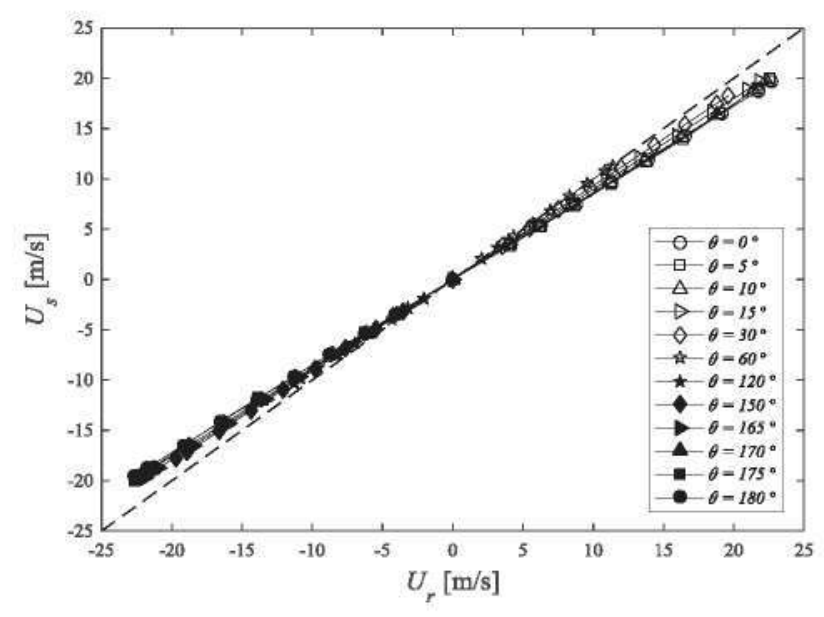

Fig. 6. Ultrasonic velocity measurements, $U_{s}$, as a function of the reference velocity, $U_{r}$

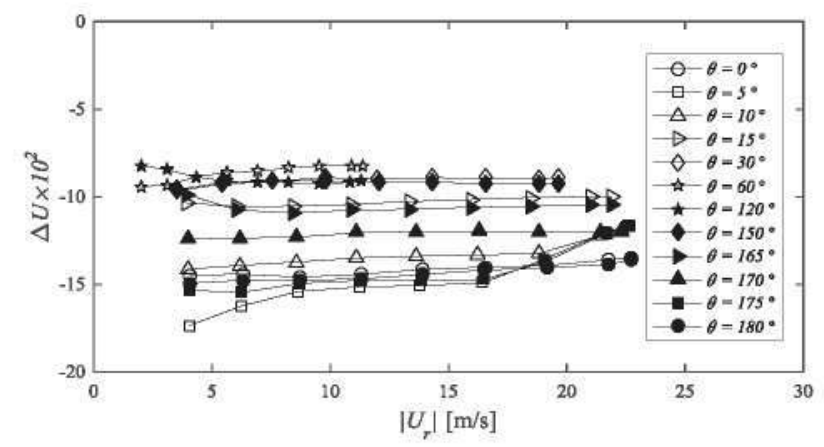

Fig. 7. Velocity deficit, $\Delta U$, as a function of the absolute value of the reference velocity, $\left|U_{\mathrm{r}}\right|$.

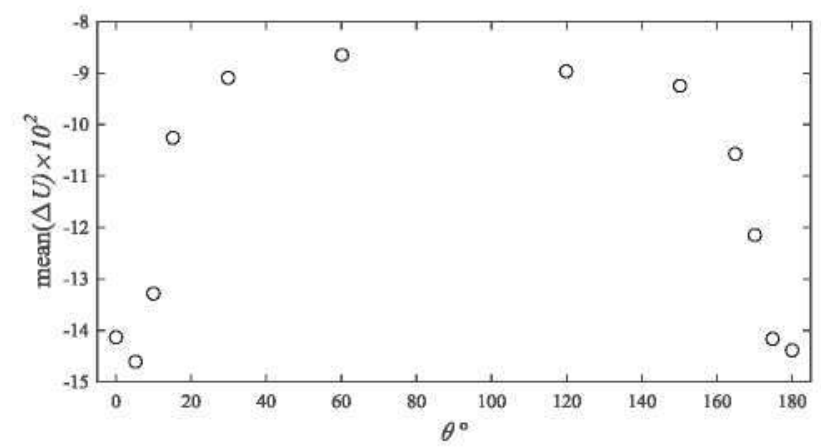

Fig. 8. Mean velocity deficit obtained for different wind speeds as a function of the orientation of the UA signal path, $\theta$.

of the free stream wind speed vector onto the signal path, $U_{r}$.

\section{The measurement uncertainties}

There are two major sources of data uncertainties in the UA measurements; the physical phenomena associated with the structure and working mechanism of the UA, and the instrumentation and systematic errors during measurement. Each one is discussed in detail in this section.

\subsection{The physical phenomena}

The transducer shadow effect is a well-known phenomenon in an ultrasonic anemometer which is the distortion on the flow along the acoustic path produced by the wake of the transducers. This leads to an underestimation in the wind velocity measured by the UA. The problem

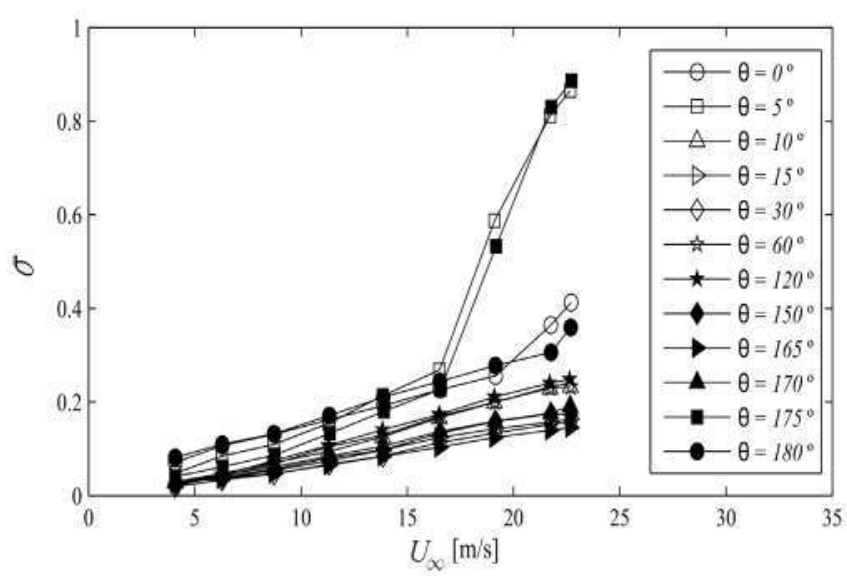

Fig. 9. Standard deviation of the ultrasonic velocity measurements, $\sigma$, as a function of the free wind speeds, $U_{\omega}$, and the orientation of the UA signal path, $\theta$.

Table 3

Calibration coefficients $A$, and $B$, for different stationary azimuthal angles, $\theta$, and coefficient of determination of the fitting, $R^{2}$.

\begin{tabular}{llll}
\hline$\theta\left[{ }^{\circ}\right]$ & $A$ & $B[\mathrm{~m} / \mathrm{s}]$ & $R^{2}$ \\
\hline 0 & 1.1531 & 0.1094 & 1.0000 \\
5 & 1.1202 & 0.4747 & 0.9996 \\
10 & 1.1331 & 0.1891 & 1.0000 \\
15 & 1.1088 & 0.0534 & 1.0000 \\
30 & 1.0729 & 0.0252 & 1.0000 \\
60 & 1.0854 & 0.0415 & 1.0000 \\
120 & 1.1048 & 0.0283 & 1.0000 \\
150 & 1.0993 & 0.0185 & 1.0000 \\
165 & 1.1184 & -0.0375 & 1.0000 \\
170 & 1.1346 & -0.0222 & 1.0000 \\
175 & 1.1241 & -0.3674 & 0.9996 \\
180 & 1.156 & -0.1325 & 1.0000 \\
\hline
\end{tabular}

can be alleviated by calibrating the anemometer or by various semiempirical correction models e.g., the one proposed by Wyngaard and Zhang [27]. They suggest that the underestimation of the measure of the wind velocity depends on the orientation of the acoustic path with respect to the wind, $\theta$, and transducer design. They use the following models for describing the directional response of the UAs:

$\frac{U_{m}}{U_{r}}=C+(1-C) \sin \theta \quad$ BAO
$\frac{U_{m}}{U_{r}}=1-(1-C) e^{-a \sin ^{2} \theta} \quad$ Kenji-Denki

$U_{m}$ is the theoretical wind speed measured by UA along the acoustic path, and $U_{r}$ is the projection of the flow vector onto that signal path, $U_{r}=U_{\infty} \cos \theta$. The constants $C$ and $a$ are the transducer shadow parameters corresponding to a given ratio of $L / D$, where $L$ is the distance between two transducers and $D$ is the diameter of the transducer.

A graph has been provided in the literature for calculating the constant $C$ (for BAO-type transducers [27] and for Kenji-Denki transducers [28]). However, the value of $a$ should be obtained from the best fitting of the theoretical model with experimental results [18].

Furthermore, a non-uniform velocity field gives rise to a shift of the otherwise straight acoustic pulse trajectory between the transmitter and receiver transducers of a sonic anemometer. This is one of the other known sources of the uncertainty in UA and has been deeply analyzed by Franchini et al. [2]. They found the correction terms for the measured wind velocity that take into account this shift in the acoustic pulse.

\subsection{The measurement errors}

Basically, the instruments and systematic errors affect the output 


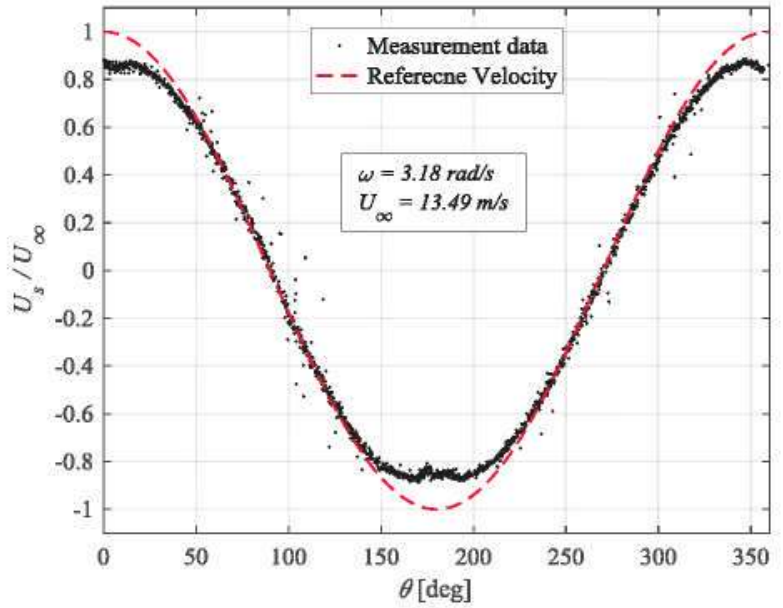

(a)

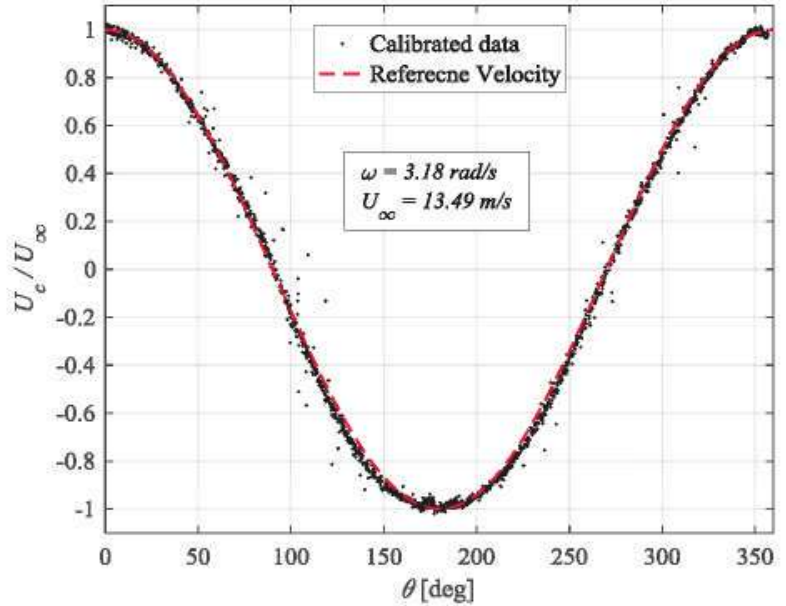

(b)

Fig. 10. Comparison of the ultrasonic velocity measurements with the reference velocities (a), and the calibrated velocity measurements with the reference velocities (b).
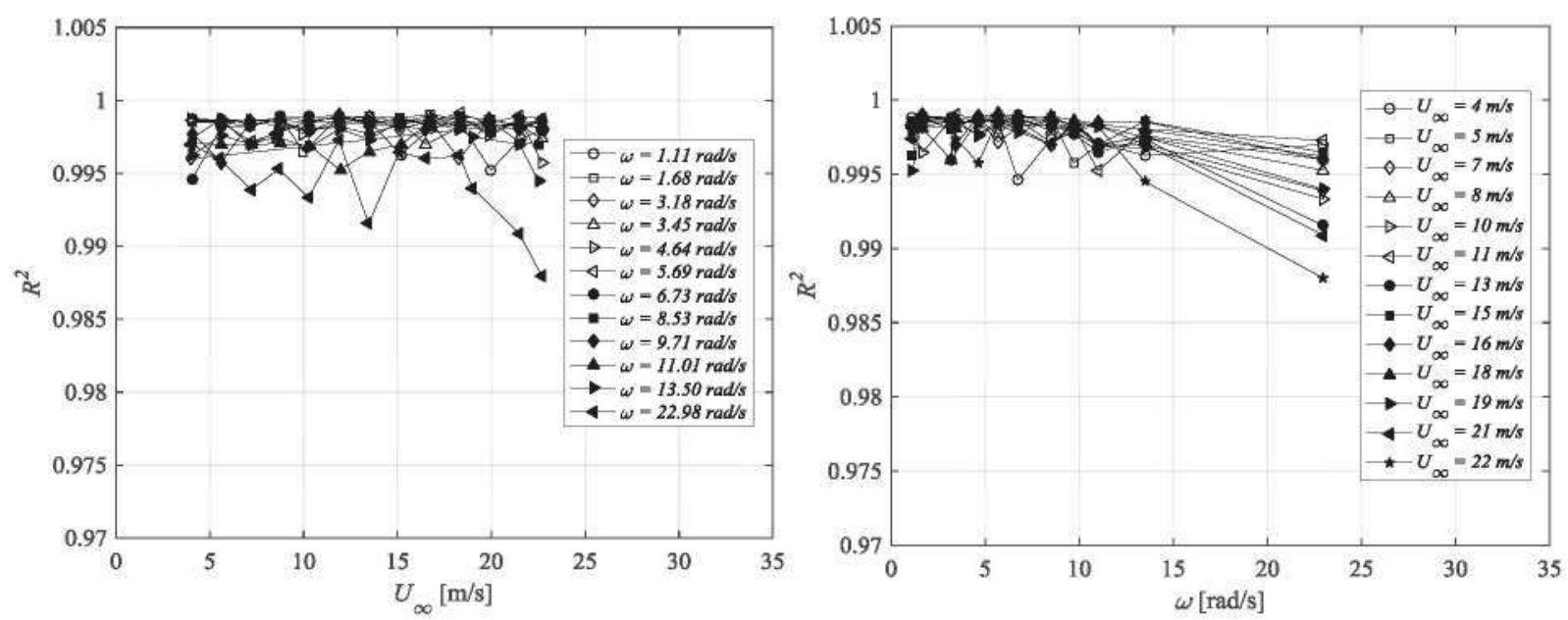

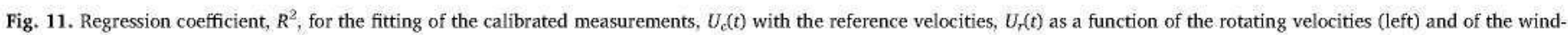
tunnel flow velocities (right).

velocity signals from the UA. Fig. 4 shows a typical dynamic test results in which, the UA was rotating at an angular velocity of $5.69 \mathrm{rad} / \mathrm{s}$ and a wind speed of $16.73 \mathrm{~m} / \mathrm{s}$. The instantaneous velocity measured by the UA is plotted for each instantaneous azimuthal angle for 10 revolutions.

Since the rotation is sinusoidal, the indicated velocity at different azimuthal angle is also of sinusoidal form except at the shadow regions, as discussed earlier. Good repeatability can be observed from this figure. However, some bad points can also be observed which can be due to UA reading and position sensor errors.

Nevertheless, the pattern must theoretically be symmetric and this is a criterion to check the reliability and repeatability of data. Data skewness, mean and median are the statistical parameters that quantitatively describe the overall behavior.

Skewness is the degree to which a data set is not symmetrical. As data becomes more symmetric, its skewness value approaches zero. The mean value describes an entire set of the measured velocities with a single value representing the center of the data. It is defined as the sum of all the measured velocities divided by the total number of samples. Median is the middle of the range of data. Half of the measured velocities are less than or equal to it and the rest are greater than or equal to it [29].

The values of these three parameters for 10 revolutions are tabulated in Table 2. Both the mean and median are nearly zero indicating that the measured velocities are symmetrically distributed between the positive and negative values corresponding to $\pi / 2<\theta<3 \pi / 2$ for negative velocities, and $3 \pi / 2<\theta<5 \pi / 2$ for positive ones. The value of the skewness is also small enough to make sure that the measured velocities are nearly symmetrical about zero mean. Histogram is a bar graph to represent the distribution of measured data [29]. The histogram of the UA measured velocity values, is shown in Fig. 5 and approves that the errors associated with the spinning mechanism, position sensor, and UA sensor are within acceptable range.

\section{The static directional calibration}

The outputs of the static tests are the free stream wind speed measured by pitot-tube, the wind speed measured by the UA for each fixed signal path direction, and the azimuthal angle, $\theta$. Fig. 6 shows the mean flow velocity measured by the UA, $U_{s}$, as a function of the reference velocity (mean wind tunnel flow velocity component along the sonic path), $U_{r}=U_{\infty} \cos \theta$, for all the tested azimuthal angles, $\theta$.

As expected, a linear relationship is observed between these two magnitudes. Nevertheless, the UA readings exhibit a certain deficit comparing to the reference velocity.

Shown in Fig. 7 is the velocity deficit, defined as $\Delta U=\left(U_{s} / U_{r}-1\right)$, as a function of the absolute value of the reference velocity, $\left|U_{r}\right|$, for the tested azimuthal angles examined. And the mean velocity deficit obtained for each reference velocity, as a function of the azimuthal angle of the ultrasonic path is shown in Fig. 8. As can be observed, for the studied range of wind speeds, $\Delta U$ is almost independent of the wind 
speed value (except in the case of $\theta= \pm 5^{\circ}$ ), while strongly depends on the orientation of the sonic path. It is seen that the velocity deficit is greater when the ultrasonic path is aligned or almost aligned with the flow.

The signature of the transducer wake on the measurements, though being beyond the scope of this paper, can be observed in this figure. As the misalignment increases, the wake of the upstream transducer is projected out of the ultrasonic path, reducing the distortion that occurs in the measurements. The study of the dependence of measured velocity-deficit with Reynolds number for small angles of incidence is left for the future research.

Fig. 9 illustrates the standard deviation, $\sigma$, of the wind speed measured by the UA for different stationary angles between signal path and wind direction. The standard deviation in the tests show a dramatic increase at $\theta=5^{\circ}$ and $\theta=175^{\circ}$ for high wind speeds. It can be reasoned that for these critical angles the upstream sensor is slightly deviated towards the wind direction so that its cross section faced up with the incoming flow increases, and therefore stronger wakes and vortices are shed into the measurement path. This underscores the role of the aerodynamic design and geometric dimensions in reducing the head shadow effect on the signal path.

Some semi-empirical models have already been proposed to correct the errors due to the aerodynamic interference effects of the sensor heads in UA wind speed measurements $[17,30]$. However, it is not possible to completely eliminate the sensor head shadow effect and the best way to reduce the uncertainty in the measurements, caused by the sensor shadow effect, is still to calibrate the anemometer.

The relationship between the wind reference velocities, projections of the incoming wind onto the signal path, and the UA indicated wind speed can be expressed as a calibration function:

$U_{r}=A U_{s}+B$

where $A$, and $B$, are the calibration coefficients which depend on the azimuthal angle of the sonic path with respect to the wind direction. The values of $A$ and $B$ are obtained by the least squares regression method and are presented in Table 3 . Also, the coefficient of determination, $R^{2}$, is included as a measure of goodness of fitting.

\section{The spinning tests}

The outputs of the tests are the wind velocity measured by the UA, $U_{s}(t)$, the instantaneous orientation of the signal path regarding the wind-tunnel flow, $\theta(t)$, and the reference wind-tunnel flow velocities, $U_{\text {os }}$, measured by pitot-tube. Since all the measurements were recorded simultaneously with the same time base, it is possible to analyze the ultrasonic measurements as a function of its orientation.

Fig. 10(a) illustrates the non-dimensional velocity measured by the UA, $U_{S}(t) / U_{\infty}$, , and the reference velocity, $U_{r}(t)$, for a typical dynamic test. Reference velocity is the ideal response that is expected to be measured by the spinning UA at each instantaneous azimuthal angles, $U_{r}(t)=U_{\infty} \cos \theta(t)$. Remarkable discrepancy in the measurements can be observed when the anemometer is crossing the azimuthal angles of $0^{\circ}, 180^{\circ}$ and $360^{\circ}$ where the transducer head distortions are at their maximum level and considerably affect the measurement path.

The calibration function already obtained from the static tests, Eq. (3), is used to correct the UA measurements in dynamic cases. The calibration coefficients, $A$ and $B$ tabulated in Table 3 , are obtained for all the instantaneous azimuthal angles, $\theta(t)$, in rotational motion and is used to correct the velocity measurements in the form of $U_{c}(t)=A(t)$ $U_{s}(t)+B(t)$

Fig. 10(b) shows the non-dimensional calibrated wind speed measurements, $U_{c}(t) / U_{\infty}$, and the reference velocity, $U_{r}(t)$, for the same presented typical test. This figure shows that the calibrated measurements are in a good agreement with the reference velocity.

To determine the accuracy of the calibration process in the spinning tests, the coefficient of determination, $R^{2}$, is calculated to indicate the performance of the calibration function, $U_{c}(t)$, in comparison with the reference velocity. Shown in Fig. 11 is the $R^{2}$ for fitting the calibrated measurements and reference velocity, for various wind speeds and UA angular velocities. Even though for high rotational velocities a slight reduction is observed in $R^{2}$, an average value of $R^{2} \approx 0.997$ is achieved throughout the test conditions and approves the high accuracy of the calibration process.

\section{Conclusions}

A modified single-axis ultrasonic anemometer was used to measure the wind speed in a subsonic wind tunnel. The experiments were performed for both static and dynamic oscillatory cases. In static tests, the velocity measured by the ultrasonic anemometer in different stationary angles of transducers signal path with respect to wind direction has been calibrated with wind-tunnel reference wind speed measured by two pitot-tubes.

The calibration coefficients obtained in the static tests were then extended to the dynamic cases where the UA was rotating with different angular velocities at various wind speeds. The calibrated measurements showed a good agreement with the reference velocity which is defined as the projection of the wind-tunnel wind speed vector onto the signal path orientation. An average regression of $R^{2}=99.7 \%$ was achieved so that the uncertainty in the dynamic tests is almost $0.3 \%$. It is observed that the measurements are affected mostly with the transducers shadow effect at each instantaneous azimuthal angle, and the shifting in acoustic pulse trajectory does not affect the UA wind speed measurements in rotational motion. Therefore, the calibration function for a fixed single-axis UA in different stationary angles of signal path with wind direction at a plane can be extended to the same anemometer in rotational motion at that plane. This procedure works for any other single axis UA as well. It must first be calibrated in various stationary yaw or pitch angles to determine the calibration constants. It has been shown that the same constants can be used to construct a correction model for the spinning case.

\section{Acknowledgement}

The work presented in this article is part of the AUM project, which is financed by the Ministry of Science and Innovation of Spain (Ministerio de Ciencia e Innovación, project reference: ENE200910670).

\section{References}

[1] A. Cuerva, A. Sanz-Andrés, J. Navarro, On multiple-path sonic anemometer measurement theory, J. Exp. Fluids 34 (2003) 345-357.

[2] S. Franchini, A. Sanz-Andres, A. Cuerva, Effect of the pulse trajectory on ultrasonic fluid velocity measurement, J. Exp. Fluids 43 (2007) 969-978.

[3] T.F. Pedersen, J. Dahlberg, A. Cuerva, F. Mouzakis, P. Busche, P. Eecen, A. SanzAndres, S. Franchini, S.M. Petersen, Accuwind - Accurate Wind Speed Measurements in Wind Energy. Summary Report. Risø National Laboratory, 2006.

[4] A. Martínez, A. Cuerva, A. Sanz-Andres, S. Franchini, The role of ultrasonic anemometry in wind energy, in: 10th German Wind Energy Conference, DEWEK2008, 2008.

[5] S. Franchini, A. Sanz-Andres, A. Cuerva, Measurement of velocity in rotational flows using ultrasonic anemometry: the flowmeter, J. Exp. Fluids 42 (6) (2007) $903-911$.

[6] E. Muramatsu, H. Murakawa, K. Sugimoto, H. Asano, N. Takenaka, N. Furuichi, Multi-wave ultrasonic Doppler method for measuring high flow-rates using stag. gered pulse intervals. J. Meas, Sci. Technol. 27 (2) (2016) 025303.

[7] M. Nabavi, K. Siddiqui, A critical review on advanced velocity measurement techniques in pulsating flows, J. Meas. Sci. Technol. 21 (4) (2010) 042002.

[8] P. Olmos, Ultrasonic velocity meter to evaluate the behavior of a solar chimney, $J$ Meas. Sci. Technol. 15 (7) (2004) N49.

[9] G.L. Pankanin, The vortex fowmeter: various methods of investigating phenomena, Meas. Sci. Technol, 16 (3) (2005) R1

[10] J.M. Wilczak, S.P. Oncley, S.A. Stage, Sonic anemometer tilt correction algorithms, Bound.-Layer Meteorol. 99 (1) (2001) 127-150.

[11] JC Wyngaard, Cup, propeller, vane, and sonic anemometers in turbulence research, Annu. Rev. Fluid Mech. 13 (1) (1981) 399-423.

[12] L. Båserud, M. Flugge, A. Bhandari, J. Reuder, Characterization of the sumo 
turbulence measurement system for wind turbine wake assessment, in: EERA Deep Wind 2014, 11th Deep Sea Offshore Wind R \& D Conference Energy Procedia, vol. 53, 2014, pp. 173-183.

[13] O.G. Castillo, E. Londner, Uav fight testing with an airborne sonic anemometer, imu and gps, in: 28th AIAA Applied Aerodynamics Conference 28 June - 1 July 2010, Chicago, Illinois, 2010.

[14] RB Mora, Flow field velocity on the flight deck of a frigate, Proc. Inst. Mech. Eng. Part G J. Aerospace Eng. (2014).

[15] A. Cuerva, A. Sanz-Andrés, Sonic anemometry of planetary atmospheres, J. Geophys. Res. 108 (E4) (2003) 5029, http://dx.doi.org/10.1029/2002JE001944

[16] D. Banfield, R.W. Dissly, Mars acoustic anemometer, in: International Workshop on Instrumentation for Planetary Missions (IPM-2012), 2012.

[17] T.F. Pedersen, N.N. Sorensen, P. Enevoldsen, Aerodynamics and characteristics of a spinner anemometer. J. Phys. Conf. Ser. 75 (2007) 012018.

[18] A. Cuerva, T.F. Pedersen, S. Luengo, J.A. Pastor, A. Sanz-Andrés, S. Franchini, A theoretical model on spinner anemometry based on ultrasonic paths, in: European Wind Energy Conference and Exhibition, 2008.

[19] T.F. Pedersen, N.N. Sorensen, L. Vita, P. Enevoldsen, Optimization of Wind Turbine Operation by Use of Spinner Anemometer, RIS $\emptyset-$ R-1654(EN), DTU, Roskilde, Denmark, 2008.

[20] F. Zahle, N.N. Sorensen, Characterization of the unsteady flow in the nacelle region of a modern wind turbine, J. Wind Energy 14 (2011) 271-283.

[21] T.F. Pedersen, Method and Apparatus to Determine the Wind Speed and Direction Experienced by a Wind Turbine, United States Patent, Patent No.: US 7,347,668 B2, 2008.

[22] L. Kristensen, Measuring high-order moments with a cup anemometer, J. Atmosph. Ocean. Technol. 17 (2000) 1139-1148.

[23] T.F. Pedersen, G. Demurtas, F. Zahle, Calibration of a spinner anemometer for yaw misalignment measurements, Wind Energy 18 (11) (2015) 1933-1952.

[24] G. Demurtas, T.F. Pedersen, F. Zahle, Calibration of a spinner anemometer for wind speed measurements, Wind Energy 19 (11) (2016) 2003-2021.

[25] Standard: IEC 61400-12-2, 2013.

[26] Report from ROMO Wind A/S, Review of the Spinner Anemometer from ROMO Wind ispin. Report No.: 113605-DKAR-R-0-1, Rev. 3, 2015.

[27] J.C. Wyngaard, S.F. Zhang, Transducer shadow effects on turbulence spectra measured by sonic anemometers, J. Atmosph. Ocean. Technol, 2 (1985) 548-558.

[28] N.G. Mortensen, Flow-Response Characteristics of the Kaijo Denki Omni-Directional Sonic Anemometer (TR-61B), RISø-R-704(EN), DTU, Roskilde, Denmark, 1994.

[29] M.J. Smith, Statistical Analysis Handbook. A Comprehensive Handbook of Statistical Concepts, Techniques and Software Tools, 2014.

[30] N.N. Sorensen, T.F. Pedersen, CFD Computation Around Wind Turbine Spinner and Nacelle, Risø-I-2579 (EN), 2007. 